Erratum

\title{
Erratum - A Novel Model for Studying Voltage-Gated Ion Channel Gene Expression during Reversible Ischemic Stroke
}

Yun-Shen Tai ${ }^{1,10}$, Shih-Chieh Yang2 ${ }^{2}$ Yi-Chun Hsieh ${ }^{3}$, Yaw-Bin Huang4,5, Pao-Chu Wu ${ }^{4}$, Ming-Jun Tsai6,7,8, Yi-Hung Tsai ${ }^{*}$, Ming-Wei Lin ${ }^{5,9 凶 * *}$

1. Department of Surgery, E-Da Hospital, Kaohsiung, Taiwan

2. Department of Orthopedic Surgery, E-Da Hospital, Kaohsiung, Taiwan

3. Department of Anesthesiology, University of Maryland School of Medicine, Baltimore, MD, USA

4. School of Pharmacy, Kaohsiung Medical University, Kaohsiung, Taiwan

5. Center for Stem Cell Research, Kaohsiung Medical University, Kaohsiung, Taiwan

6. Department of Neurology, China Medical University Hospital, Taichung, Taiwan

7. School of Medicine, China Medical University, Taichung, Taiwan

8. Department of Neurology, China Medical University, An-Nan Hospital, Tainan, Taiwan

9. Department of Medical Research, E-Da Hospital/ E-Da Cancer Hospital, Kaohsiung, Taiwan

10. I-Shou University College of Medicine, Kaohsiung, Taiwan

*Equal contributors

$\triangle$ Corresponding author: Ming-Wei Lin, Department of Medical Research, E-Da Hospital/ E-Da Cancer Hospital, Kaohsiung 824, Taiwan. TEL: 886-7-6151100 ext.5413. E-mail: ta990074@gmail.com

(C) The author(s). This is an open access article distributed under the terms of the Creative Commons Attribution License (https://creativecommons.org/licenses/by/4.0/). See http://ivyspring.com/terms for full terms and conditions.

Published: 2019.09.18

Corrected article: Int J Med Sci. 2019; 16(1): 60-67. doi: 10.7150/ijms.27442.

The first author's affiliation should be corrected by adding "I-Shou University College of Medicine, Kaohsiung, Taiwan" (Affiliation No. 10), to the "Department of Surgery, E-Da Hospital, Kaohsiung, Taiwan." (Affiliation No. 1), as shown in the title page above.

\section{References}

1. Tai YS, Yang SC, Hsieh YC, Huang YB, Wu PC, Tsai MJ, Tsai YH, Lin MW. A Novel Model for Studying Voltage-Gated Ion Channel Gene Expression during Reversible Ischemic Stroke. Int J Med Sci 2019; 16(1):60-67. doi:10.7150/ijms.27442. 